\title{
Peroral Endoscopic Myotomy for the Treatment of Achalasia in a 10-Year-Old Male Patient
}

\author{
Jörg Filser ${ }^{1}$ Anke Dick ${ }^{2}$ Thomas Meyer ${ }^{1} \quad$ Christoph-Thomas Germer ${ }^{1}$ Burkard H.A. von Rahden ${ }^{1}$
}

${ }^{1}$ Department of General, Visceral, Vascular and Paediatric Surgery,
University Medical Center, Würzburg, Germany
2 University Children's Hospital, University Medical Center,
Würzburg, Germany

Eur J Pediatr Surg Rep 2015;3:18-22.
Address for correspondence Jörg Filser, Department of General, Visceral, Vascular and Paediatric Surgery, University Medical Center, Oberdürrbacherstrasse 6, Würzburg 97080, Germany (e-mail: jfilser@googlemail.com).
Abstract
Keywords
- achalasia
- POEM
- peroral endoscopic myotomy

Peroral endoscopic myotomy (POEM) is a new endoscopic treatment for achalasia with very good short-term results in adults. Data about POEM in pediatric patients are missing. We present the case of a 10-year-old male patient with type I (classic) achalasia, successfully treated with POEM. The procedure was accomplished in a similar fashion to the technique used in adults. Short-term results were fine, with a complete control of dysphagia and absence of reflux. We suggest that POEM is a suitable option in pediatric patients-similar to adults-but long-term results must be awaited.

\section{Introduction}

Achalasia is a rare disease with an incidence of 1 per 100,000 patients in adults. ${ }^{1}$ The disease also occurs in childrenthrough all age groups-however, it is estimated even less frequent in the pediatric population ( 0.11 cases per 100,000 patients ${ }^{2}$ ). Achalasia is a rare esophageal motility disorder characterized by failure of the lower esophageal sphincter (LOS) to relax and aperistalsis of the tubular esophagus. Pneumatic dilation (PD) and laparoscopic Heller myotomy (LHM) are well established and currently used as standard treatment for achalasia in children., ${ }^{3,4}$ Peroral endoscopic myotomy (POEM) is a new, entirely endoscopic procedure for the treatment of achalasia. Myotomy is performed exclusively through the transoral endoscopic route and is therefore a true NOTES procedure (Natural Orifice Endoscopic Surgery). Current data regarding POEM in adults demonstrate excellent postoperative outcome. $^{5-7}$ Data about POEM in pediatric patients are still missing.

\section{Case Report}

We present here the case of a 10 -year-old male patient admitted to our department with an 18-month history of dysphagia, recurrent vomiting, and weight loss. The patient complained about regurgitations appearing immediately after food intake. General pediatric workup including gastroscopy showed no morphologic correlative for these symptoms. High-resolution manometry (HRM)/esophageal pressure topography (EPT) study using Sierra (Sierra Scientific Instruments Inc., Los Angeles, California, United States), according to the standards defined in the Chicago classification ${ }^{8}$ was performed to check for esophageal motility disorder. EPT data were analyzed using ManoView analysis software (Given Imaging Ltd., Yokneam, Israel). This investigation revealed an increased integrated relaxation pressure (IRP) of the LOS $(15.5 \mathrm{~mm} \mathrm{Hg}$ ) and aperistalsis of the tubular esophagus, with $100 \%$ simultaneous (tertiary) contractions ( - Fig. 1). According to the Chicago classification of esophageal motility disorders, this result had to be classified as type I (classic) achalasia. $^{8}$

Additional timed-esophagography (Fluoro Loop [3/s; Global Siemens Healthcare, Erlangen, Germany] and fluoroscope with last-image-hold-technique) showed a dilated esophagus with slow transit of the contrast medium across the esophagogastric junction (OeGJ) (-Fig. 2). The Eckardt dysphagia score ${ }^{9}$ was 8 points (weight loss 1 ; dysphagia 3 ; retrosternal pain 1 ; and regurgitation 3 ).

The different available treatment options were discussed with the parents of the patient. Due to the convincing results
License terms

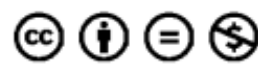

received

November 6, 2013 accepted after revision

January 26, 2014

published online

July 17,2014
DOI http://dx.doi.org/

10.1055/s-0034-1372461. ISSN 2194-7619. (c) 2015 Georg Thieme Verlag KG Stuttgart · New York 


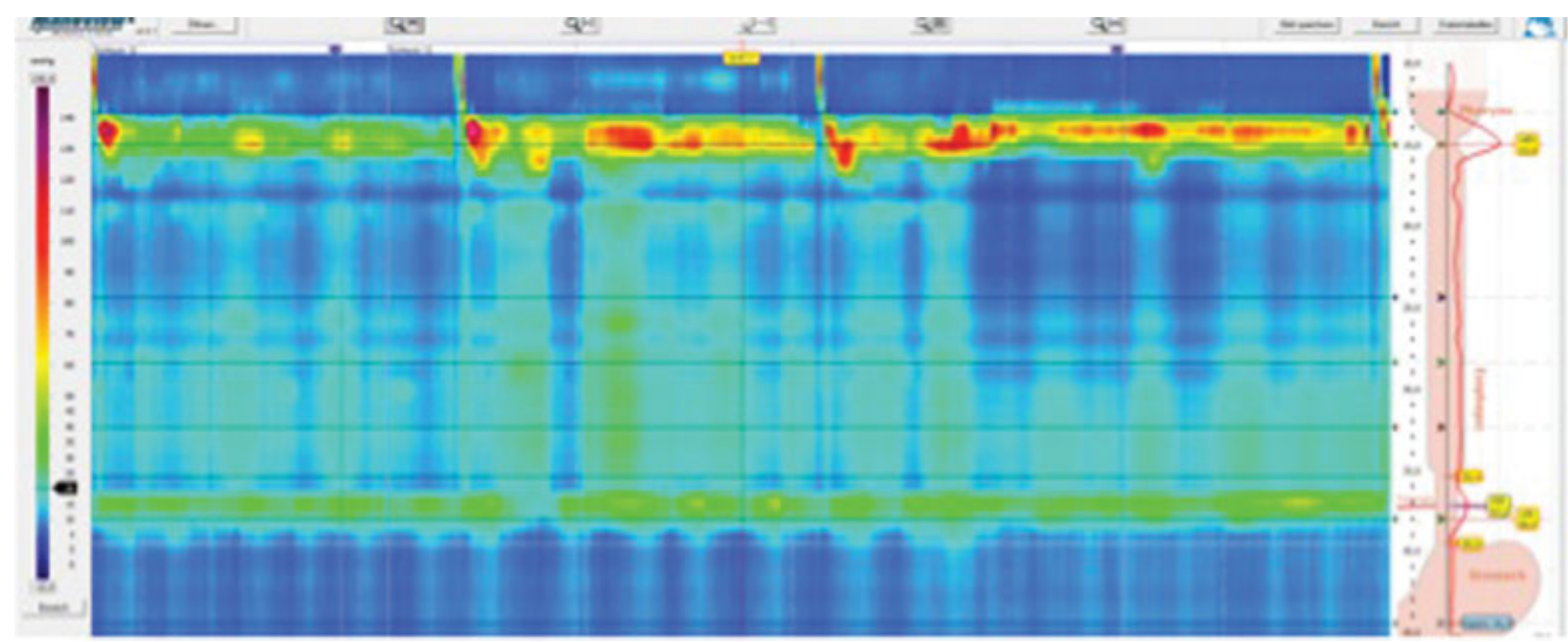

Fig. 1 HRM/EPT study shows aperistalsis of the tubular esophagus with $100 \%$ of the contractions being simultaneous (tertiary) contractions. HRM, high-resolution manometry; EPT, esophageal pressure topography.

of POEM in adults-in our own experience as well as the available literature ${ }^{5-7}$-we decided to offer the POEM procedure.

The POEM procedure was performed using the technique suggested by Inoue et al as follows ${ }^{5}$ : (1) creation of a mucosal entry, (2) creation of a submucosal tunnel using an endoscopic submucosal dissection technique, (3) myotomy, and (4) clip closure of the mucosal entry (-Fig. 3A-E).

For this procedure we used the "Silverscope" by KARL STORZ, Tuttlingen, Germany, type 13821PKS, $9.3 \mathrm{~mm}$. The whole procedure was performed using carbon oxide insufflation (UCR, Olympus Medical Systems Corp., Tokyo, Japan). A cap attachment with straight orifice (DH28GR, Fujifilm, Tokyo, Japan) was used for all steps of the procedure (submucosal tunnel, myotomy). Closure of the mucosal incision, was

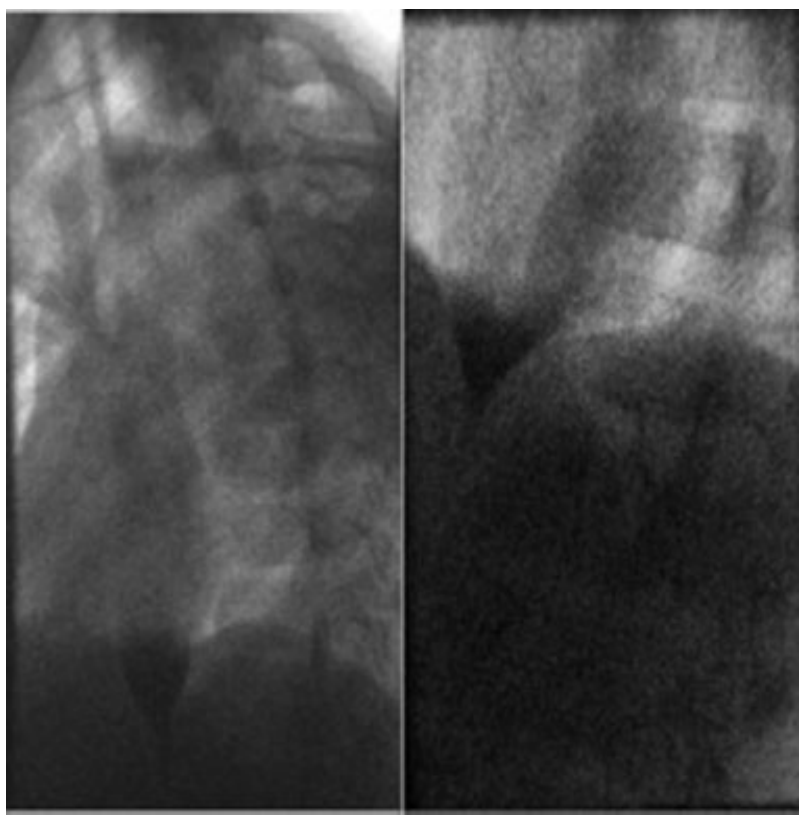

Fig. 2 Preoperative timed esophagography. performed with an oblique cap attachment. The POEM procedure was carried out under general anesthesia with the patient in supine position. Submucosal injection of $10 \mathrm{~mL}$ indigocarmine-stained saline solution, which was applied with a special injection device (InjectorForceMax, NM401L-0425, Olympus) was performed $2 \mathrm{~cm}$ below the aortic arch (clearly visible at $30 \mathrm{~cm}$ from the incisors). The mucosal entry was created with the triangle tip (TT)-knife (KD-640L; Olympus) and the energy source VIO300D (ERBE Elektromedizin GmbH; Tübingen, Germany) in "cut" mode (80 W; effect 2).

Dissection of the submucosal space was also performed with the TT-knife. Stained saline solution was repeatedly injected into the submucosal space, using a blunt catheter (PW-205V; Olympus) and dissection was performed with the energy source in "spray" mode (50 W; effect 2 ). The submucosal tunnel was created in the tubular esophagus and carried forward across the OeGJ (35 cm below the dental line). Myotomy of the circular muscle fibers was started approximately $3 \mathrm{~cm}$ below the mucosal entry and conducted across the OeGJ until $2 \mathrm{~cm}$ at the gastric site $(31-37 \mathrm{~cm}$ below the dental line), whereas the longitudinal muscle layer was preserved to serve as "safety margin."

For clip closure of the mucosal entry, we used a cap attachment with oblique orifice (MH-588; Olympus). Seven endoclips (HX-610-090L; Olympus) were applied with the standard multiuse clip applicator (HX110QR; Olympus). The operating time was 93 minutes.

Postoperatively the patient was allowed to drink small sips of water. Proton pump inhibitors (PPI) as well as cefuroxime were administered intravenously for 3 days and then switched to per oral. Postoperatively, a slightly elevated C-reactive protein levels $(3.6 \mathrm{mg} / \mathrm{dL})$ with normal white blood cell were noted. On postoperative day 1 a timed esophagography was performed, which demonstrated prompt delay of the esophagus (-Fig. 4) and no signs of leakage. After an unremarkable postoperative course, the patient was discharged home on day 3 in good general condition with 

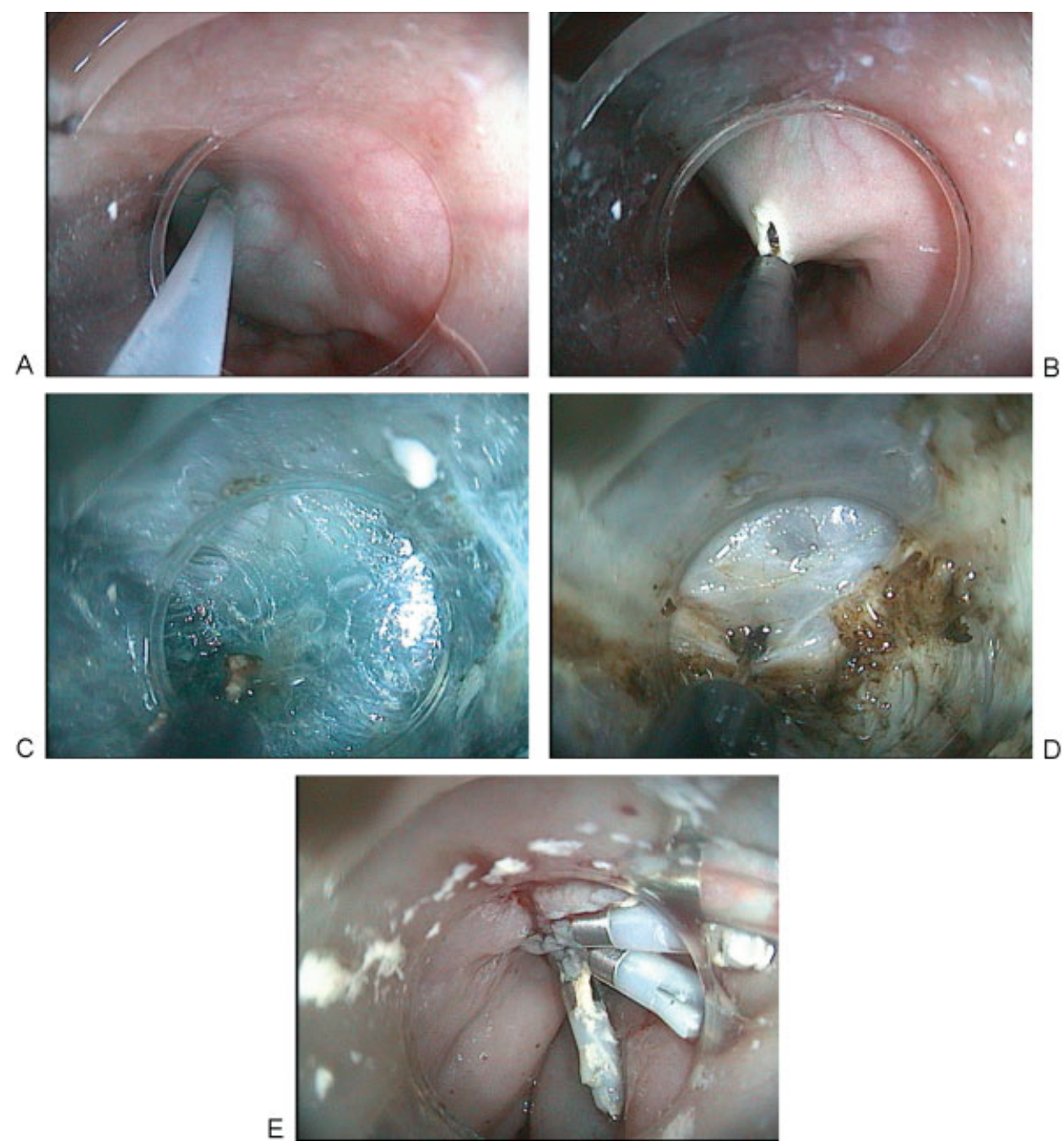

Fig. 3 (A) Mucosal injection; (B) mucosal incision; (C) submucosal dissection, using an ESD-technique, with repeated injection of indigocarminestained solution and dissection using TT knife. (D) Endoscopic myotomy of circular muscle fibers, using the TT knife; (E) clip closure. ESD, endoscopic submucosal dissection; TT, triangle tip.

very good symptom relief, that is, absence of dysphagia, and no clinical reflux symptoms. Short-term follow-up (2 months) showed no clinical impairment with complete control of dysphagia (Eckardt score 0 ) and no sign of reflux.

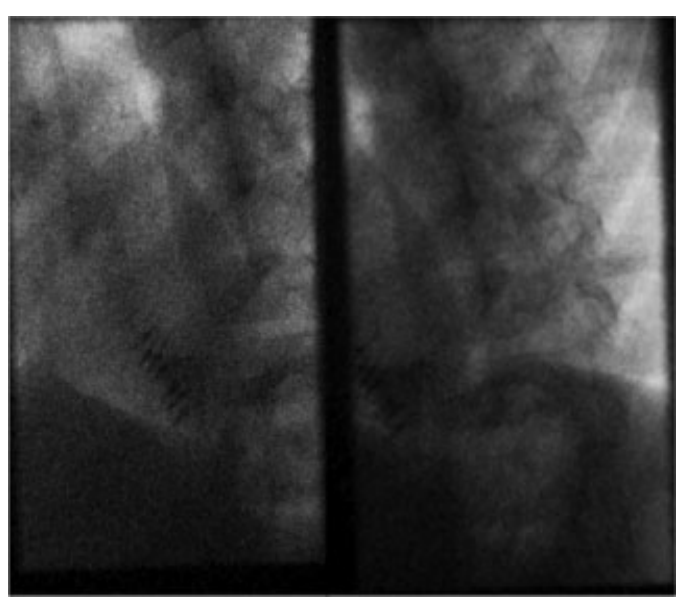

Fig. 4 Postoperative timed esophagography.

\section{Discussion}

POEM is a promising new technique for treatment of achalasia, with more than 1,400 cases having been performed in specialized centers worldwide to date. ${ }^{6,10}$ The procedure is attractive due to the myotomy being performed entirely through the endoscopic route and its excellent results, that is, good control of dysphagia and low reflux rates in the short term. However, little is known about the potential role of POEM for treatment of pediatric achalasia patients. Only two previous reports have addressed this issue: Maselli et al have reported briefly about a successfully accomplished POEM procedure in a 3-year-old achalasia patient with growth retardation and Down syndrome. ${ }^{11}$ Familari et al reported about successful POEM procedures in three female achalasia patients $(9,9$, and 11 years of age) with no intra- and postoperative complications and complete symptom relief (normalized Eckardt score) at 1-year follow-up. ${ }^{12}$ These reports as well as our experience suggest that POEM might be well suited as treatment option for pediatric achalasia patients as well. Due to our excellent results with POEM in adult achalasia patients, we decided to recommend this well-suited 
technique for our pediatric patient. At this time we had an experience of altogether 11 POEM procedures performed in adults. Before introducing POEM in clinical practice we had undergone a special training in Yokohama/Japan and had trained the procedure in a well-suited animal model. Therefore, we had discussed the circumstances, a new procedure without long-term experience and rather no experience in children, but excellent short-term results in adults with the parents, who agreed to have POEM done on their child.

Technical aspects as well as the procedure itself did not differ from our experience in adults. Anatomical landmarks were identified easily, especially the OeGJ, which is a crucial landmark for this procedure, and the impression of the aortic arch. To confirm a save intragastric position, we followed the suggestions made by Inoue et $\mathrm{al}^{5}$ which are also nicely summarized in the IPOEM (International Per Oral Endoscopic Myotomy) survey by Stavropulos et $\mathrm{al}^{6}$ : the myotomy has completely addressed the muscle responsible for achalasia, when the endoscope reaches a wide submucosal space with larger vessels. Further indicators are detection of indigocarmine stain in retroflexed view, level from the incisors and smooth passage of the endoscope through the previously tight OeGJ.

As a new procedure for the treatment of achalasia POEM must exhibit some outstanding characteristics to be a serious alternative to the current procedures PD and LHM in children. The free choice of length and localization of the myotomy may be regarded as a fundamental advantage of the POEM procedure. A longer myotomy may be beneficial in patients suffering from chest pain, and perhaps especially in patients with type III achalasia, where PD and LHM are potentially inferior.

POEM could also be an option for esophageal motility disorders in which pharmacological treatment has failed. ${ }^{13}$ As a pure NOTES procedure the manipulation in the abdominal cavity as well as visible scars are avoided. This could be a weighty argument for this procedure referring to the future body image of adolescents.

Also, the previous operations in the abdominal cavity are assessed as a reasonable indication for POEM. ${ }^{6,14,15}$ But which side effects should be mentioned particularly in the consultation with patient and the parents? The focus of interest should be the potential appearance of gastroesophageal reflux after POEM.

Despite all the promising early experiences with POEM, some aspects warrant controversial discussion. One of these is the exact rate of postoperative reflux, which is currently not known. One might expect a higher reflux rate after POEM compared with LHM, because the myotomy is not combined with a fundoplication, which is standard when performing LHM. ${ }^{16,17}$ However, an antireflux procedure might-on the other hand-be unnecessary after POEM, because natural antireflux barriers such as the phrenoesophageal membrane and the angle of His remain untouched. The current available literature is controversial regarding reflux rates after POEM, ranging from 5.9 to $46 \% .^{5,7}$ The overall reflux rates of 20 to $30 \%$ are probably the correct estimate ${ }^{10}$ and are pretty similar to with reflux rates after LHM and PD. ${ }^{17,18}$ Most reported reflux-associated problems are minor complaints, which are well treatable with PPI. Nevertheless, this issue will be of the special interest because of the longer exposition of the (distal) esophagus to reflux in children compared with adults.

One other aspect regarding differences between POEM and LHM is the type of myotomy: during LHM, all muscle layers (circular and longitudinal) are cut. During POEM we cut only the circular muscle fibers, whereas the longitudinal muscle is preserved, following the suggestion by Inoue et al. $^{5} \mathrm{~A}$ recent randomized study from China ${ }^{19}$ has shown, that there is no difference with respect to inclusion or exclusion of longitudinal fibers in the myotomy. This supports the current view of protagonists of the POEM procedure, that only the circular muscle layer is responsible for achalasia.

All currently available publications reported no serious local infections although the setting of this procedure would suggest this. To reduce the potential risk of infection we perform an application of a topical antibiotic (gentamycin) and administer intravenous antibiotics for 1 week postoperatively.

If $\mathrm{CO}_{2}$ insufflation is used ${ }^{20}$ our experience as well as reported data revealed predominantly minor complications such as pneumoperitoneum, cutaneous emphysemas, pneumothorax, which are well treatable intraoperative or are mostly self-limitated. 5,7,14,20,21 Mucosal lesions, which were normally depict and provide at the postoperative endoscopy cannot be regularly identified in our described setting. Therefore, other strategies have to be developed to prevent serious events as described above. The risk of major bleeding especially in the submucosal tunnel seems to be low but also difficult to manage. ${ }^{22}$

One final issue to discuss is the question what to do after a potential treatment failure of POEM. Again all three options for treatment of achalasia (LHM, PD, and POEM) may be considered: (1) LHM might be an option but might well be more difficult, because there might be severe scarring in the submucosal tunnel and at the outer surface of the mucosa, which might show stronger adherence to the muscular layers. (2) PD might also be an option. However, the risk for esophageal perforation might be higher after POEM, when the muscular layer at the esophagogastric junction is already weakened. (3) The best option in this setting might again be a (redo) POEM procedure. This could be performed at the posterior esophageal wall, which is untouched at this time. Only recently, Inoue et al have reported POEM as a redo procedure after failed myotomy. ${ }^{23}$

\section{Conclusion}

Technical aspects of the POEM procedure in children do not differ from adults. Pre- and postoperative treatment concepts must be tailored to children conditions and detailed information of the parents is crucial. In an adjusted setting, POEM seems to be a suitable alternative for the treatment of achalasia. Larger case series and long-term follow-up are required.

Conflict of Interest

None. 


\section{References}

1 Mayberry JF. Epidemiology and demographics of achalasia. Gastrointest Endosc Clin N Am 2001;11(2):235-248, v

2 Hussain SZ, Thomas R, Tolia V. A review of achalasia in 33 children. Dig Dis Sci 2002;47(11):2538-2543

3 Di Nardo G, Rossi P, Oliva S, et al. Pneumatic balloon dilation in pediatric achalasia: efficacy and factors predicting outcome at a single tertiary pediatric gastroenterology center. Gastrointest Endosc 2012;76(5):927-932

4 Lee CW, Kays DW, Chen MK, Islam S. Outcomes of treatment of childhood achalasia. J Pediatr Surg 2010;45(6):1173-1177

5 Inoue $\mathrm{H}$, Minami $\mathrm{H}$, Kobayashi $\mathrm{Y}$, et al. Peroral endoscopic myotomy (POEM) for esophageal achalasia. Endoscopy 2010;42(4):265-271

6 Stavropoulos SN, Modayil RJ, Friedel D, Savides T. The International Per Oral Endoscopic Myotomy Survey (IPOEMS): a snapshot of the global POEM experience. Surg Endosc 2013;27(9):3322-3338

7 Swanstrom LL, Kurian A, Dunst CM, Sharata A, Bhayani N, Rieder E. Long-term outcomes of an endoscopic myotomy for achalasia: the POEM procedure. Ann Surg 2012;256(4):659-667

8 Bredenoord AJ, Fox M, Kahrilas PJ, Pandolfino JE, Schwizer W, Smout AJ; International High Resolution Manometry Working Group. Chicago classification criteria of esophageal motility disorders defined in high resolution esophageal pressure topography. Neurogastroenterol Motil 2012;24(Suppl 1):57-65

9 Eckardt VF. Clinical presentations and complications of achalasia. Gastrointest Endosc Clin N Am 2001;11(2):281-292, vi

10 Swanstrom LL. Poetry is in the air: first multi-institutional results of the per-oral endoscopic myotomy procedure for achalasia. Gastroenterology 2013;145(2):272-273

11 Maselli R, Inoue H, Misawa M, et al. Peroral endoscopic myotomy (POEM) in a 3-year-old girl with severe growth retardation, achalasia, and Down syndrome. Endoscopy 2012;44(2, Suppl 2 UCTN) E285-E287

12 Familiari P, Marchese M, Gigante G, et al. Peroral endoscopic myotomy for the treatment of achalasia in children. J Pediatr Gastroenterol Nutr 2013;57(6):794-797

13 Shiwaku H, Inoue H, Beppu R, et al. Successful treatment of diffuse esophageal spasm by peroral endoscopic myotomy. Gastrointest Endosc 2013;77(1):149-150
14 Sharata A, Kurian AA, Dunst CM, Bhayani NH, Reavis KM, Swanström LL. Peroral endoscopic myotomy (POEM) is safe and effective in the setting of prior endoscopic intervention. J Gastrointest Surg 2013;17(7):1188-1192

15 Zhou PH, Li QL, Yao LQ et al. Peroral endoscopic remyotomy for failed Heller myotomy: a prospective single-center study. Endoscopy 2013;45(3):161-166

16 Richards WO, Torquati A, Holzman MD, et al. Heller myotomy versus Heller myotomy with Dor fundoplication for achalasia: a prospective randomized double-blind clinical trial. Ann Surg 2004;240(3):405-412, discussion 412-415

17 Campos GM, Vittinghoff E, Rabl C, et al. Endoscopic and surgical treatments for achalasia: a systematic review and meta-analysis. Ann Surg 2009;249(1):45-57

18 Boeckxstaens GE, Annese V, des Varannes SB, et al; European Achalasia Trial Investigators. Pneumatic dilation versus laparoscopic Heller's myotomy for idiopathic achalasia. N Engl J Med 2011; 364(19):1807-1816

19 Li QL, Chen WF, Zhou PH, et al. Peroral endoscopic myotomy for the treatment of achalasia: a clinical comparative study of endoscopic full-thickness and circular muscle myotomy. J Am Coll Surg 2013; 217(3):442-451

20 Ren Z, Zhong Y, Zhou P, et al. Perioperative management and treatment for complications during and after peroral endoscopic myotomy (POEM) for esophageal achalasia (EA) (data from 119 cases). Surg Endosc 2012;26(11):3267-3272

21 Von Renteln D, Fuchs KH, Fockens P, et al. Peroral endoscopic myotomy for the treatment of achalasia: an international prospective multicenter study. Gastroenterology 2013;145(2):309-311, e1-e3

22 Li QL, Zhou PH, Yao LQ, et al. Early diagnosis and management of delayed bleeding in the submucosal tunnel after peroral endoscopic myotomy for achalasia (with video). Gastrointest Endosc 2013;78(2):370-374

23 Onimaru M, Inoue $\mathrm{H}$, Ikeda $\mathrm{H}$, et al. Peroral endoscopic myotomy is a viable option for failed surgical esophagocardiomyotomy instead of redo surgical Heller myotomy: a single center prospective study. J Am Coll Surg 2013;217(4):598-605 